\title{
A Latino Patient-Centered, Evidence-Based Approach to Diabetes Prevention
}

\author{
Lisa G. Rosas, PhD, MPH, Nan Lv, PhD, Megan A. Lewis, PhD, \\ Elizabeth M. Venditti, PhD, Patricia Zavella, PhD, Veronica Luna, BS, \\ and Jun $M a, M D, P h D$
}

Introduction: Cultural tailoring of evidence-based diabetes prevention program (DPP) interventions is needed to effectively address obesity and its related chronic diseases among Latinos in primary care. This article describes the patient-centered process used to adapt the DPP and reports cultural adaptations.

Methods: We used a 2-stage formative research process to culturally adapt an evidence-based DPP intervention in the context of primary care. The first stage involved 5 focus groups of Latino patients and interviews with 5 stakeholders ( 3 with primary care physicians and 2 with medical directors) to inform a first round of adaptations. The second stage included pretesting the stage I-adapted intervention with a Latino patient advisory board to complete a second round of adaptations.

Results: Key stakeholders involved in this 2-stage adaptation process included 34 Latino patients who participated in 5 focus groups and 5 physicians and medical directors who participated in key informant interviews during stage I and 11 patients who attended the 16 advisory board meetings and their family members who attended 1 of the meetings during stage II. Using this patient-centered stakeholder-engaged approach, we found the original intervention was largely congruent with the cultural values of the study population. To further strengthen the cultural relevance of the intervention, salient cultural values emphasized by patients and stakeholders underscored the importance of family and community support for behavior change. Accordingly, key adaptations were made to (1) invite family members to the orientation session and at 2 other key timepoints to facilitate family support, (2) provide participants support from the coach and each other via smartphone applications, and (3) provide healthy, easy, low-cost culturally appropriate meals at each group session.

Conclusions: The 2-stage approach actively engaging patients, family members, providers, and health care system leaders reinforced the cultural congruence of the existing intervention while further strengthening it with adaptations promoting Latino family and community support. ( $\mathrm{J}$ Am Board Fam Med 2018;31:364-374.)

Keywords: Chronic Disease, Diabetes Mellitus, Focus Groups, Hispanic Americans, Medical Directors, Obesity, Primary Care Physicians, Smartphone

Over the past 15 years, the prevalence of obesity has increased among Latinos and the disparity with non-Hispanic whites (NHW) has widened in the

This article was externally peer reviewed.

Submitted 5 July 2017; revised 3 January 2018; accepted 6 January 2018.

From Stanford University, CA (LGR); University of Illinois at Chicago, IL (NL, JM); RTI International, Research Triangle Park, North Carolina, WA (MAL); University of Pittsburgh, PA (EMV); University of California, Santa Cruz (PZ); Palo Alto Medical Foundation (VL).

Funding: This project was supported by grant R01HS022702 from the Agency for Healthcare Research and Quality and internal funding from the Palo Alto Medical Foundation Research Institute. The content is solely the responsibility
United States. ${ }^{1,2}$ Currently, $43 \%$ of Latinos are obese versus $36 \%$ of NHWs. In addition, Latinos are less likely than NHWs to engage in healthy lifestyle behaviors, such as consuming fruits and

of the authors and does not necessarily represent the official views of the Agency for Healthcare Research and Quality. No sponsor or funding source had a role in the design or conduct of the study; collection, management, analysis or interpretation of the data; or preparation, review or approval of the manuscript.

Conflict of interest: none declared.

Corresponding author: Lisa G. Rosas, PhD, MPH, Department of Health Research and Policy and Primary Care and Population Health, 1070 Arastradero Rd., Palo Alto, CA 94304 (E-mail: lgrosas@stanford.edu). 
vegetables and engaging in regular physical activity. ${ }^{3-5}$ As a result, Latinos are $50 \%$ more likely to suffer from higher rates of obesity- and lifestylerelated chronic disease such as diabetes. ${ }^{6}$ Given that Latinos currently represent $17 \%$ of the US population and are expected to grow to $29 \%$ by 2060, culturally tailored interventions to address obesity and promote healthy lifestyles are urgently needed. ${ }^{7}$

We and others have published robust evidence from randomized controlled trials (RCTs) for the effectiveness of practical lifestyle interventions for weight management and cardiometabolic risk reduction in primary care. ${ }^{8-10}$ Initially, the landmark Diabetes Prevention Program (DPP) efficacy trial demonstrated that an intensive one-on-one behavioral lifestyle intervention targeting moderate weight loss and increased physical activity conferred 58\% lower incidence of diabetes than placebo in high-risk patients. Translation research has extended this evidence to demonstrate the effectiveness of group-based (and other forms of) DPP. Based on this evidence, the US Preventive Services Task Force recommends lifestyle counseling for high-risk adults in primary care settings ${ }^{11}$, and the Center for Medicare and Medicaid Services now reimburses primary care-based behavior therapy for obesity ${ }^{12}$ and for weight management and reduction of cardiometabolic risk ${ }^{13}$, making primary care an ideal setting for implementing behavioral lifestyle interventions. In addition, weight management programs supplementing face-to-face intervention with technology-mediated ongoing coach contact and support have also shown significant positive benefits. ${ }^{14,15}$

However, the majority of studies on lifestyle interventions have enrolled largely NHW samples; a systematic review revealed that $71 \%$ of participants in DPP translation studies were NHW. ${ }^{16}$ Latino culture differs from mainstream American culture in several domains that may be important for behavioral lifestyle interventions, such as social networks, family structure, understandings of heredity of disease, acceptable means of risk communication, and barriers to resource access. ${ }^{17-21}$ For example, familismo, which refers to the value placed on the immediate and extended family, may be important to address as part of the intervention. ${ }^{20,21}$ Furthermore, no DPP translation studies, especially technology-supported DPP, with this priority population have been conducted in pri- mary care, leaving a critical evidence gap for Latinos. ${ }^{7,22}$ Latinos have increasing access to primary care $^{23}$, which can be an effective context to promote sustainable chronic disease prevention. Thus, cultural adaptation of evidence-based technologysupported DPP translations is needed to effectively address obesity and its related clinical sequelae among Latinos in primary care.

To ensure that interventions reflect Latino cultural preferences, direct engagement of patients, providers, and other key stakeholders is fundamental. ${ }^{7,24}$ The Patient-Centered Outcomes Research Institute's (PCORI) Patient Engagement Rubric calls for research that involves patients and their stakeholders in all phases of the research process, including in formative research aimed at adapting interventions for specific settings and populations. ${ }^{25}$ Such stakeholder-engaged approaches are likely to become increasingly important given the diversification of the US population; by 2055 the US will not have a single racial or ethnic majority. ${ }^{26}$ However, research that actively engages Latino patients and providers in a systematic process to culturally adapt an evidence-based behavioral lifestyle intervention is limited.

Based on the PCORI Engagement Rubric, we used a patient-centered iterative process to adapt a DPP-based intervention for Latinos in primary care as part of the Vida Sana study. The dual goals of this article are to describe the patient-centered process used to adapt an evidence-based translation of the DPP in primary care and to report on the resulting adaptations.

\section{Methods}

We used the PCORI Patient Engagement Rubric to guide engagement with Latino patients, their family members, providers, and health care system leaders for the adaptation process (Table 1). The goal was to adapt the group lifestyle balance (GLB) intervention to meet Latinos' cultural preferences and needs. The culturally adapted intervention will be tested in a RCT compared with usual care in a primary care setting; the RCT protocol has been previously published. ${ }^{27}$

\section{Group Lifestyle Balance (GLB)}

We chose the GLB program for adaptation due to robust evidence supporting its effectiveness and existing infrastructure to promote dissemination. 
Table 1. Description of Patient-Centered Outcomes Research Institute Patient Engagement Rubric with Examples from the Study

\begin{tabular}{ll}
\hline Engagement Principle & Example from Study \\
\hline $\begin{array}{l}\text { Reciprocal relationships: Roles of researchers, patients, } \\
\text { and stakeholders are determined collaboratively and } \\
\text { clearly stated. }\end{array}$ & $\begin{array}{c}\text { Latino patient advisory board (LPAB) was formed with the } \\
\text { goal of adapting the Group Lifestyle Balance intervention. }\end{array}$ \\
$\begin{array}{ll}\text { Colearning: Researchers, patients, and other } \\
\text { stakeholders share their expertise with each other } \\
\text { for mutual benefit. }\end{array}$ & $\begin{array}{l}\text { The first LPAB meeting included a discussion of roles, } \\
\text { responsibilities, and ground rules. }\end{array}$ \\
& - Focus groups were used for researchers to learn from patients \\
& about their experience. \\
& The LPAB provided their expertise in the cultural adaptation \\
process. & - Researchers shared their expertise in behavioral interventions \\
$\begin{array}{l}\text { Partnerships: Time and contributions of patients and } \\
\text { stakeholders are valued and demonstrated in fair } \\
\text { financial compensation. Also refers to a commitment } \\
\text { to diversity and cultural competence. }\end{array}$ & $\begin{array}{l}\text { with the LPAB during the adaptation process. } \\
\text { Transparency, honesty, and trust: Information is } \\
\text { readily shared among all partners. }\end{array}$ \\
\hline
\end{tabular}

The effectiveness of the GLB program has been demonstrated in various settings, including primary care by our team and others ${ }^{8,28-33}$, and it is recognized by the Centers for Disease Control and Prevention's National DPP. ${ }^{34}$ If proven effective, the adapted intervention can be rapidly disseminated through the University of Pittsburgh Diabetes Prevention Support Center whose goal is to support the dissemination of the GLB intervention.

GLB program is based on Social Cognitive Theory ${ }^{35}$, which emphasizes a reciprocally determined relationship between the individual, environment, and behavior. Based on this theoretic basis, the GLB program uses a goal-based approach to promote positive outcome expectancies and foster selfefficacy by targeting at least $7 \%$ weight loss and a minimum of 150 minutes per week of moderateintensity physical activity. It uses a lifestyle coachled group format and has 2 distinct periods over 1 year: (1) intensive treatment (core) and (2) maintenance support (postcore). The intensive treatment period includes 12 weekly core sessions followed by 4 biweekly core transition sessions, for a total of 16 sessions delivered within the first 6 months of the program. The postcore period includes an additional 6 monthly sessions covering topics such as how to prepare for long-term self-management, mindful eating, and stress management for a total duration of 12 months. All the GLB participant handouts were previously translated into Spanish ${ }^{36}$, which was a fundamental step in adapting lifestyle interventions for Latinos. ${ }^{37}$ However, the GLB program had not been specifically adapted to Latino culture before this study.

\section{Adaptation Process}

The Vida Sana formative research phase used a 2-stage process to culturally adapt the GLB intervention for Latino patients in primary care (Figure 1). The first stage involved focus groups with $\mathrm{La}-$ tino patients and interviews with primary care physicians and health care system leaders (medical directors) to inform a first round of adaptations. The second stage included pretesting the adapted intervention from stage I with a Latino patient advisory board and making a second round of adaptations based on their review and input.

\section{Stage I}

To engage Latino patients, we conducted 5 focus groups (4 to 10 participants per group) until we reached saturation in themes (ie, point at which no

Figure 1. Cultural adaptation process for Vida Sana intervention.

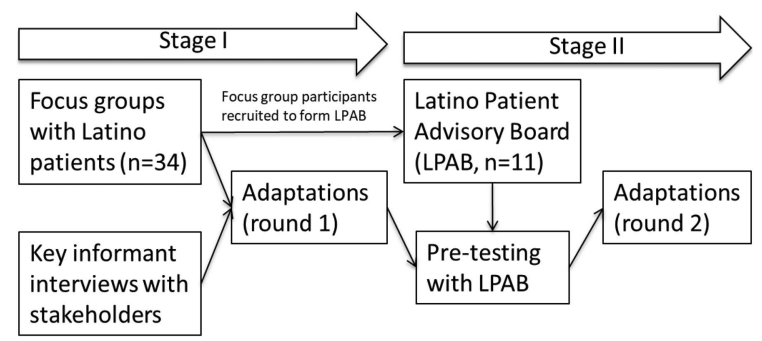


new themes emerge). ${ }^{38}$ Participants were primary care patients from a large community-based multispecialty group practice in Silicon Valley, California. We recruited Latino adults with a body mass index $>24 \mathrm{~kg} / \mathrm{m}^{2}$ and who had prediabetes ${ }^{39}$, a history of gestational diabetes, and/or metabolic syndrome, ${ }^{40}$ but not type 1 or type 2 diabetes or cardiovascular disease. Patients whose electronic health record documented the above criteria received recruitment invitations by email or mail (if no email address in the electronic health record). The objectives of the focus groups were to determine (1) the influence of culture on health and weight; (2) the perceived causes, prevalence, and impact of obesity and related lifestyle risk factors; (3) awareness of and attitudes toward weight management and risk-factor prevention strategies; (4) facilitators and barriers to a healthy lifestyle; and (5) acceptance and use of technology. During one of the groups, participants completed usability testing of the FitBit wireless activity monitors and MyFitnessPal website and mobile application for tracking weight, dietary intake and physical activity. FitBit and MyFitnessPal were chosen because of their accessibility in Spanish and options for social networking.

We conducted key-informant interviews with 3 providers whose practice panels included large numbers of Latino patients and 2 health care system leaders (Medical Directors) who are important for future implementation. The purpose of the interviews was to understand their perceptions of the local Latino population's needs for weight control and diabetes prevention and how programs could be effective, generalizable, and sustainable. For example, questions included the following: (1) What are some of your patients' facilitators and barriers to weight loss, specifically with the Latino population? (2) Are there any interventions or strategies that you have utilized that seem to work better with the Latino population? (3) What are your perceived barriers/challenges to managing overweight/obese patients? (4) How would a program like this to-beadapted intervention fit into your practice?

The research team members reviewed focus group recordings and notes to summarize patient feedback according to the 5 focus group objectives. Subsequently, the research team identified common themes relevant for adaptation across the 5 objectives. Disagreements were solved through group discussion. The same method was applied to summarize the themes for relevant adaptation from the key stakeholder interviews. Based on the practical goals of adapting the intervention, no further qualitative analysis was conducted. All intervention materials were reviewed to incorporate these themes wherever possible. In addition to incorporating changes, the research team highlighted key topics to address in the subsequent adaptation stage, including any salient cultural values that were highlighted by focus group and key stakeholder interview participants.

\section{Stage II}

To further culturally adapt the GLB program, we invited 26 focus group participants who expressed interest in further involvement in the project to join a Latino patient advisory board. A bilingual and bicultural research assistant with no prior health education or lifestyle coaching qualifications received a 1-week training by a GLB master trainer to serve as the lifestyle coach. The lifestyle coach delivered the 22 sessions that had been adapted in stage I to the advisory board over 16 weekly meetings. Family members of the patient advisors were invited to one meeting, session 7 of the GLB program focused on problem solving. Using existing major topics within each session, the coach stopped after each topic within the session and asked advisory board members to fill out a semistructured survey in which they evaluated cultural appropriateness and relevance. The survey used Likert scales from 1 (not culturally appropriate or relevant) to 5 (highly culturally appropriate and relevant) and open-ended questions to probe other suggested changes to the content and delivery. To ensure the cultural appropriateness, the coach facilitated a discussion to adapt any topic where at least one board member recorded a score of 3 or lower on cultural appropriateness or relevance. Board members were also asked to provide recommendations for changes related to presentation or examples provided in the materials (eg, whether the food examples were culturally relevant). ${ }^{17,18}$

\section{Results}

\section{Stage I}

Focus group participants $(n=34)$ were primarily foreign born $(85 \%)$ of Mexican descent with a mean age at migration of $23 \pm 9.3$ years and mean 
Table 2. Focus Group Participant Characteristics $(\mathrm{N}=34)$

\begin{tabular}{|c|c|c|}
\hline Characteristic & $\begin{array}{l}\mathrm{n} \text { or } \\
\text { mean }\end{array}$ & $(\%$ or $\mathrm{SD})$ \\
\hline Female, n (\%) & 19 & $(56)$ \\
\hline \multicolumn{3}{|l|}{ Nativity, n (\%) } \\
\hline US born & 5 & $(15)$ \\
\hline Foreign born, Mexico & 22 & $(65)$ \\
\hline Foreign born, other & 7 & $(20)$ \\
\hline $\begin{array}{l}\text { Years in the US (foreign born), } \\
\text { mean (SD) }\end{array}$ & 24 & $(10.6)$ \\
\hline Age at migration, mean (SD) & 23 & $(9.3)$ \\
\hline \multicolumn{3}{|l|}{ Education, n (\%) } \\
\hline Less than high school & 3 & (9) \\
\hline High school graduate & 9 & $(27)$ \\
\hline Some college & 8 & (24) \\
\hline College or more & 13 & (39) \\
\hline \multicolumn{3}{|l|}{ Monthly household income, n (\%) } \\
\hline Less than $\$ 3,000$ & 7 & $(21)$ \\
\hline$\$ 3,000-\$ 6,999$ & 5 & $(15)$ \\
\hline$\$ 7,000$ or more & 7 & $(21)$ \\
\hline Household members, mean (SD) & 4 & (1.4) \\
\hline \multicolumn{3}{|l|}{$\begin{array}{l}\text { Language preference for written } \\
\text { materials, } n(\%)\end{array}$} \\
\hline Spanish & 21 & $(62)$ \\
\hline English & 5 & $(15)$ \\
\hline $\begin{array}{l}\text { No preference for Spanish or } \\
\text { English }\end{array}$ & 8 & (24) \\
\hline \multicolumn{3}{|l|}{$\begin{array}{l}\text { Language preference for speaking, } \\
\text { n (\%) }\end{array}$} \\
\hline Spanish & 21 & (62) \\
\hline English & 1 & (3) \\
\hline No preference & 12 & (36) \\
\hline
\end{tabular}

SD, standard deviation.

years of US residence of $24 \pm 10.6$ years (Table 2). The educational level of participants varied from high school education or less (36\%), some college education (24\%), to a college degree or more (39\%). The majority of participants preferred Spanish for both written and oral communication (62\%). For written communication, $15 \%$ preferred English and 24\% reported no preference. For oral communication, $36 \%$ reported no preference between English and Spanish and only 3\% preferred English.

Table 3 lists the themes that emerged from the focus groups that are relevant to intervention adaptation. Based on these themes, the most important stage 1 adaptations made to the delivery of the intervention were to invite family members to 3 key intervention sessions to facilitate their supporting role, offer a healthy meal at each session, emphasize the use of the FitBit activity tracker early on and throughout, including the social networking options, and provide individualized feedback to participants based on the self-monitoring data provided via the FitBit and MyFitnessPal. Notably, participants who pretested the FitBit and MyFitnessPal applications confirmed that they were accessible in Spanish, could provide key features such as social networking, and in the case of MyFitnessPal, were able to track culturally specific foods (eg, enchiladas and tacos).

Stage 1 adaptations also pertained to the content and meaning of the intervention session materials. These changes addressed participant feedback on healthy eating patterns and physical activity components. For example, the term diet ("dieta" in Spanish) was removed and replaced with the term for food ("alimentación" in Spanish). The word diet was understood to have a negative connotation implying overly restrictive eating patterns that were not favored by participants nor reflective of intervention goals. For physical activity, one adaptation was made in response to participants' discussion of how lifestyles have become increasingly sedentary with increased time in the US. Some participants attributed this to a shift from occupations that include more activity (eg, agricultural or service/ hospitality jobs) to occupations that are primarily office-based and include few opportunities for activity. To address this, we highlighted the existing GLB content on the mixture of planned and unplanned behavior and how this may differ for each participant depending on their occupation. Minor adaptations included adding culturally specific Latino food and activity examples on several participant handouts.

Stage 1 participants were also specifically queried on their preference for participating in an intervention with Latinos from diverse backgrounds or with Latinos of shared cultural ancestry. Participants focused their discussion on the perceived benefits of engaging in discussions about diet and physical activity with Latinos from diverse countries of origin including the US, Mexico, and Central and South America. The primary benefit mentioned was the opportunity to learn about foods and activities from Latinos with different backgrounds from their own. The Spanish language was viewed as a unifying feature more important than any differences. When probed about 
Table 3. Feedback from Latino Focus Group Participants and Adaptations in Stage I

Focus Group Theme Category $\quad$ Focus Group Feedback

1st Round of Adaptations

Family

Community

Cultural foods patterns

Physical Activity

Tech use
- Family was a key component of culture.

- Family support was most commonly reported as positive influence for changing lifestyle behaviors.

- Negative influences from families included family members who were unsupportive of lifestyle changes or weight goals; time pressure/competing priorities from family members.

- Community was a key component of Latino culture.

- Food was an important element of Latino culture.

- Cultural foods were the most commonly reported negative influences on healthy eating.

- Traditional Latino eating patterns of a larger lunch and a smaller dinner were thought to promote weight maintenance.

- Negative connotation of the term "diet" associated with restriction, deprivation, and limited food variety.

- Challenges to healthy eating included eating breakfast consistently; finding time to cook appealing meals for the whole family.

- Physical activity in groups and children were sources of motivation to make healthy changes.

- Increased time in the US was associated with less occupational physical (more sedentary jobs) activity and exercise.

- Dancing and soccer were commonly mentioned as physical activity options that were culturally congruent. Three types of physical activity were discussed:

$\circ$ Exercise: Zumba, walking, hiking, soccer, and gym workout.

o Occupational: jobs in restaurants, hospitals, housekeeping and childcare.

○ Daily living: housekeeping, gardening, and commuting.

- Heterogeneous familiarity with self-tracking via smartphone or web-based applications.

- Most participants were able to easily use the smartphone and web-based MyFitnessPal application to track diet. All but one participant downloaded the application on a smartphone without assistance.

- All are interested in using the FitBit activity monitor as part of the intervention.

- The MyFitnessPal application was available in both Spanish and English, which was very helpful.
- Included family members at 3 key timepoints in the intervention: the orientation session, a midintervention session, and the last session for graduation (Delivery).

- Developed tips for being a supportive family member (Content).

- Provided support for participants to connect via the Smartphone applications (Delivery).

- Included potluck meals (Delivery).

- Incorporated a healthy meal at every group session (Delivery).

- Ensured that meals provided healthy versions of cultural foods; examples included "sopes*," ceviche, and black bean soup (Delivery).

- Included more Latino food examples in intervention materials, including healthier, notso-healthy, and traditional foods (Content).

- The traditional Latino eating pattern was included as one option for a healthy eating pattern (Content).

- Avoided the term "diet" in favor of terms such as nutrition, eating, and eating plan (Content).

- Designed all session meals to account for budgets, time in preparation, and cultural norms (Delivery).

- Provided time for physical activity in groups during the sessions and encouraged physical activity among group participants outside of sessions (Content).

- Highlighted the distinction between leisuretime and occupational physical activity options and that each participants' activity mixture may be made up of different levels of each (Content).

- Emphasized the use of the pedometer to capture all types of physical activity (leisuretime, occupational, and daily living) (Delivery).

- Included more culturally congruent physical activity examples such as dancing and soccer (Content).

- Allowed for time to introduce and troubleshoot the smartphone applications (Content).

- Provided training on skills for self-tracking early in the intervention (Delivery).

- Intervention coach provided online and inperson feedback on self-tracking information (Delivery).

- Provided all participants with a FitBit (Delivery).

- Encouraged participants to use MyFitnessPal application in their preferred language (Delivery).

*Sope is a traditional Mexican appetizer that includes vegetable and/or meat toppings on a thick corn meal base. 
potential disadvantages, no participants discussed any drawbacks.

The 5 stakeholder interviews were completed with 2 family medicine providers, 1 internal medicine provider, and 2 health care system leaders (medical directors). All respondents vocalized a strong need for programs culturally adapted for Latino patients and that they lacked appropriate programs and materials to offer their Latino patients with obesity. They also mentioned that it was important to consider the heterogeneity of Latino patients in adapting the intervention. One provider mentioned that her Latino patients differed greatly in terms of income, education, occupation, and number of years in the US. Health care system leadership felt that interventions culturally tailored for Latinos were an important strategy for providing patient-centered care. Stakeholders did not provide specific suggestions for the adaptation of delivery or content of the intervention.

\section{Stage II}

Following focus groups, 15 participants were available to join the Latino patient advisory board to further adapt the intervention. Four participants were unable to attend following the first or second meeting, leaving 11 Latino patient advisory board members. Attendance over 16 advisory board meetings was high, with an average of at least 9 participants $(85 \%)$ at each meeting. Participants were offered a healthy meal at each meeting and asked to provide feedback on whether the meal should be offered for future groups. Participants eagerly connected via the FitBit application and engaged in physical activity with other members outside of meetings, suggesting that group cohesion was an important factor.

Board members found the stage I-adapted GLB intervention to be generally acceptable and congruent with Latino cultural values. They discussed that having a bilingual/bicultural Latino coach and other Latino participants was the most influential factor in making the intervention feel culturally relevant to them. They also expressed that the intervention had ample opportunities for tailoring to the diversity within the Latino patient population, given the use of individualized feedback based on self-tracking of diet and physical activity. Finally, board members discussed the importance of other qualities of the lifestyle coach apart from being bilingual/bicultural, including being a good facilitator, providing quality individualized feedback, and the ability to connect individually with the diversity of participants.

Despite an overall acceptance of the intervention, the advisory board recommended several key adaptations for the core sessions (sessions 1 to 12; see Table 4): (1) Add an orientation session before session 1 to provide a brief program overview and include family members to ensure that family members understood the intervention, its goals, and simple tips for providing support from the outset; (2) Incorporate the " $\mathrm{My}$ Plate" visual in the orientation, as it was helpful for communicating the types of food choices recommended in the program; and (3) Invite family members to session 6 and 12 (instead of session 7 on problem solving) and include a potluck. Session 6 was chosen because of the focus on environmental determinants in which family member support was seen as critical. Session 12 was chosen to emphasize the importance of family member support, as the session frequency transitions from weekly to biweekly and monthly.

Other adaptations to the core sessions that the advisory board recommended were to (1) provide training on self tracking of physical activity and diet in session 1 to capitalize on early motivation; (2) further emphasize the distinction between occupational and leisure time physical activity as it relates to planned and unplanned activity; and (3) add a discussion of how to handle social gatherings in a culturally congruent way to session 8 on eating out. The advisory board did not recommend significant adaptations for the postcore sessions (sessions 13 to 22).

\section{Discussion}

Latino patients, their family members, providers, and medical directors were actively involved in a 2 -stage process to culturally adapt an evidencebased behavioral lifestyle intervention for Latino patients in primary care. Using this patient-engaged approach, we found that the GLB program was largely congruent with Latino cultural values, while we also identified specific opportunities to adapt the intervention to further ensure cultural appropriateness for diverse Latino patients in primary care. The adaptations to the intervention content primarily included providing cultural food, physical activity, and social gathering strategy examples, time for physical activity in session, highlights on leisure-time and occupational physical 
Original Group Lifestyle

Balance Session Pretest Feedback

2nd Round of Adaptations

Session 1: Welcome and getting started losing weight
- Add an orientation session to the program and include family members so that they understand the intervention, its goals, and have simple tips for providing support to the participant.

- Introduce skills sooner to capitalize on motivation.

Session 2: Be a fat and calorie detective

Session 3: Healthy eating

Session 4: Move those muscles

- Very dense and long, consider reducing and moving some information to other session.

- Introduce MyPlate earlier because it is important for calorie counting context and the visual is helpful for changing food choices.

- The session is too long.

- Positive feedback overall.

- Positive feedback overall.

Session 5: Tip the calorie balance

Session 6: Take charge of what's around you

Session 7: Problem solving

Session 8: Four keys to healthy eating out

Session 9: Slippery slope of lifestyle change

Session 10: Jump start your activity plan

Session 11: Make social cues work for you

Session 12: Ways to stay motivated
- Positive feedback overall.

- The inclusion of family members was very positive but wanted them to be included in the prior session.

- Positive feedback overall.

- Positive feedback overall.

- Introduce physical activity monitor earlier in session 1 because it allows participants to communicate via the smartphone application outside of the group sessions and it was helpful for tracking physical activity from the beginning.

- Positive feedback overall.

- Positive feedback overall.
- Added an orientation session to accomplish the goals suggested by the patient advisors. The MyPlate graphic and concept is used to explain the intervention dietary goals to participants and their family members in the orientation session (Content).

- Provided training on self-tracking diet and physical activity in session 1 (Content).

- Moved a detailed section on weighing and measuring to session 1 to provide participants with tracking skills early on (Content).

- Moved up the section on MyPlate to the orientation session (Content).

- Moved discussion on pedometer from session 10 to this session (Content).

- Further emphasized the distinction between leisure-time and occupational physical activity as they relate to planned versus unplanned activity (Content).

- No changes.

- Invited family members to this session (Delivery)

- Included a potluck to create a sense of community with family members (Delivery).

- Family members were added to session 6 (Delivery).

- Discussed strategies for social gatherings that are culturally congruent (Content).

- No changes.

- Moved up the introduction of the pedometer to the first session (Content).

- Added a group walk to promote a sense of community (Content).

- No changes.

- Invited family members to session as a celebration for graduation; this included a second potluck where participants are encouraged to bring a healthy dish (Delivery). activity, and adding an orientation session to describe the intervention. The most important adaptations to the delivery were to (1) invite family members to the orientation session and 2 other key timepoints to facilitate family support, (2) provide participants support from coach and each other via smartphone applications, and (3) provide healthy, easy, economic, cultural meals at each group session.

This study fills a critical gap in research aimed at culturally tailoring behavioral lifestyle interventions for Latinos in primary care. A recent system- 
atic review identified 12 culturally tailored diabetes prevention interventions for Latinos and none were based in primary care. ${ }^{7}$ Primary care is an ideal setting for implementing behavioral lifestyle interventions for all patients, including Latinos. Latinos increasingly have access to primary care ${ }^{23}$, and primary care leverages the weight of providers' recommendations, provides opportunities for ongoing support for maintaining behavior change, and can be integrated with management of comorbid conditions. Given increasing policy support for providing DPP-based interventions in primary care, culturally adapting these programs is critical for addressing disparities in obesity and obesity-related chronic conditions including diabetes. ${ }^{1,6,41}$

Directly engaging patients and key stakeholders was successful for adapting an evidence-based behavioral lifestyle intervention. The adaptations identified through this process have also been recommended in previous cultural adaptations of behavioral lifestyle interventions. ${ }^{7}$ For example, offering traditional foods and recipes and including family members has been used in previous cultural adaptations. ${ }^{7}$ Other common adaptations, such as modifying materials for low literacy level, offering the intervention in a community setting, and using community health workers, have been used in the past but did not emerge in this study. It is possible that literacy issues were not discussed because the GLB program was already designed at fifth-grade reading level. Although focus group participants and advisory board members were not asked to give input on the setting, no one discussed any drawbacks to offering the intervention in the primary care setting. This may reflect the fact that patients were recruited through the primary care setting. Providers and medical directors were highly supportive of providing the intervention in primary care. Finally, participants in focus groups and advisory board members were highly supportive of having a trained lifestyle coach who has many similarities with a community health worker, including sharing a cultural background, speaking Spanish, and having an understanding of participants' social and environmental context.

In this study, the use of technology including the FitBit activity tracker and MyFitnessPal was feasible and acceptable among middle age Latinos with varying levels of familiarity with technology. The technologies enabled personalized feedback and promoted group cohesion, both of which were identified as key strategies for engaging Latinos in a behavioral lifestyle intervention. Emerging studies also find Latinos can effectively use technology tools to improve health behaviors such as physical activity. ${ }^{42-44}$ In addition, current national data ${ }^{45-47}$ show that Internet and mobile technology access and use for health are comparable, and sometimes even greater, among Latinos than NHWs, and continued penetration of technology is expected. Although a segment of the Latino population does not currently have access to the Internet and/or mobile technology, this should not be a barrier to developing the evidence base for health interventions using these technologies. If not addressed, that would only accentuate the digital health divide for this already disadvantaged population.

It is important to note that this study has some potential limitations. Mainly, the adaptation reflects the cultural perspectives of the Latino patients, providers, and key stakeholders that were included in the process. Nationally, the Latino population is highly heterogeneous, and Latino subgroups may differ from each other in their culture values as well as their perspectives on lifestyle behaviors. For example, cultural values may differ according to country of origin, gender, age, and other factors. In addition, behaviors such as diet and physical activity are known to differ according to nativity and length of residence in the US with those born in the US and with longer US residence known to have less healthy diet and physical activity patterns compared with those who are foreign born and who have lived in the US for less time. ${ }^{48-50}$ Our focus group participants were primarily middle-aged, foreign born, and of Mexican descent. However, representatives of various important subgroups including men, immigrants from Central America (represented in the "other" category in Table 1), and US-born Latinos were included, and their views are reflected in the adapted intervention. The small sample size precluded summarizing feedback by these demographic characteristics. Groups that seek to implement the culturally adapted intervention can undertake additional tailoring to customize the intervention for important subgroups.

In conclusion, Vida Sana's systematic and participatory approach enabled infusing Latino cultural worldviews, beliefs, values, and behaviors into the existing evidence-based GLB intervention. The process demonstrates the feasibility of engaging patients, their family members, and providers di- 
rectly in the research process, as described in PCORI's Patient Engagement Rubric. An ongoing RCT will provide insight into the effectiveness of this adapted version of the GLB program among Latino patients in a primary care setting. ${ }^{27}$ If proven effective and feasible, the program may have the potential for wide-spread positive impact through implementation in primary care settings.

The authors extend special thanks to Vida Sana participants and their families who make this study possible. The authors also thank Lily Liang, MPH, for her contributions to the synthesis of results and writing of the manuscript.

To see this article online, please go to: http://jabfm.org/content/ 31/3/364.full.

\section{References}

1. Ogden CL, Carroll MD, Kit BK, Flegal KM. Prevalence of obesity among adults: United States, 20112012. NCHS Data Brief 2013;131:1-8.

2. Fryar CD, Carroll MD, Ogden CL. Prevalence of overweight, obesity, and extreme obesity among adults: United States, 1960-1962 through 20112012. Centers for Disease Control and Prevention. 2014. Available from: https://www.cdc.gov/nchs/ data/hestat/obesity_adult_11_12/obesity_adult_ 11_12.htm.

3. August KJ, Sorkin DH. Racial/ethnic disparities in exercise and dietary behaviors of middle-aged and older adults. J Gen Intern Med 2011;26:245-50.

4. Epstein JF, Sugerman SB, Mitchell P, Induni $M$. Prevalence of fruit and vegetable consumption and physical activity by gender and race/ethnicity-California, 2005-2006. Calif J Health Promot 2008;6: 61-72.

5. Centers for Disease Control and Prevention. Prevalence of fruit and vegetable consumption and physical activity by race/ethnicity-United States, 2007. Available from: https://www.cdc.gov/mmwr/preview/ mmwrhtml/mm5613a2.htm.

6. Centers for Disease Control and Prevention. Hispanic health: Preventing type 2 diabetes. 2016. Available from: https://www.cdc.gov/features/hispanichealth/ index.html. Accessed on June 28, 2017.

7. McCurley JL, Gutierrez AP, Gallo LC. Diabetes prevention in U.S. Hispanic adults: A systematic review of culturally tailored anterventions. Am J Prev Med 2017;52:519-29.

8. Ma J, Yank V, Xiao L, et al. Translating the Diabetes Prevention Program lifestyle intervention for weight loss into primary care: A randomized trial. JAMA Intern Med 2013;173:113-21.

9. Appel LJ, Clark JM, Yeh HC, et al. Comparative effectiveness of weight-loss interventions in clinical practice. N Engl J Med 2011;365:1959-68.
10. Wadden TA, Volger S, Sarwer DB, et al. A two-year randomized trial of obesity treatment in primary care practice. N Engl J Med 2011;365:1969-79.

11. LeFevre ML, Force USPST. Behavioral counseling to promote a healthful diet and physical activity for cardiovascular disease prevention in adults with cardiovascular risk factors: U.S. Preventive Services Task Force recommendation statement. Ann Intern Med 2014;161:587-93.

12. Center for Medicare and Medicaid Services. Decision memo for intensive behavioral therapy for obesity (CAG-00423N). 2011. Available from: https:// www.cms.gov/medicare-coverage-database/details/ nca-decision-memo.aspx? \&NcaName = Intensive $\%$ 20Behavioral\%20Therapy\%20for\%200besity $\& b c=$ ACAAAAAAIAAA $\& N C A I d=253$. Accessed on June 28, 2017.

13. Spitalnic P. Certification of Medicare Diabetes Prevention Program. Baltimore, MD: Department of Health and Human Services; 2016.

14. Arem H, Irwin M. A review of web-based weight loss interventions in adults. Obes Rev 2011;12:e236-243.

15. Krebs P, Prochaska JO, Rossi JS. A meta-analysis of computer-tailored interventions for health behavior change. Prev Med 2010;51:214-221.

16. Ali MK, Echouffo-Tcheugui J, Williamson DF. How effective were lifestyle interventions in realworld settings that were modeled on the Diabetes Prevention Program? Health Aff (Millwood) 2012; 31:67-75.

17. Castro FG, Barrera M, Jr., Martinez CR, Jr.. The cultural adaptation of prevention interventions: Resolving tensions between fidelity and fit. Prev Sci 2004;5:41-5.

18. Resnicow K, Soler R, Braithwaite RL, Ahluwalia JS, Butler J. Cultural sensitivity in substance use prevention. J Community Psychol 2000;28:271-90.

19. Rosal MC, Borg A, Bodenlos JS, Tellez T, Ockene IS. Awareness of diabetes risk factors and prevention strategies among a sample of low-income Latinos with no known diagnosis of diabetes. Diabetes Educ 2011;37:47-55.

20. Perez-Stable E. Issues in Latino health care. West J Med 1987;146:213-8.

21. Elder JP, Ayala GX, Parra-Medina D, Talavera GA. Health communication in the Latino community: Issues and approaches. Annu Rev Public Health 2009;30:227-51.

22. Marquez B, Anderson A, Wing RR, et al. The relationship of social support with treatment adherence and weight loss in Latinos with type 2 diabetes. Obesity (Silver Spring) 2016;24:568-75.

23. Chen J, Vargas-Bustamante A, Mortensen K, Ortega AN. Racial and ethnic disparities in health care access and utilization under the Affordable Care Act. Med Care 2016;54:140-6.

24. Wallerstein N, Duran B. Community-based participatory research contributions to intervention re- 
search: the intersection of science and practice to improve health equity. Am J Public Health 2010; 100:S40-46.

25. Patient-Centered Outcomes Research Institute. Engagement Rubric for Applicants. 2016. Available from: http://www.pcori.org/sites/default/files/EngagementRubric.pdf. Accessed June 28, 2017.

26. Cohn D, Caumont A. 10 demographic trends that are shaping the U.S. and the world. 2016. Available from: http://www.pewresearch.org/fact-tank/2016/ 03/31/10-demographic-trends-that-are-shaping-theu-s-and-the-world/. Accessed June 23, 2017.

27. Rosas LG, Lv N, Xiao L, et al. Evaluation of a culturally-adapted lifestyle intervention to treat elevated cardiometabolic risk of Latino adults in primary care (Vida Sana): A randomized controlled trial. Contemp Clin Trials 2016;48:30-40.

28. Kramer MK, Miller RG, Siminerio LM. Evaluation of a community Diabetes Prevention Program delivered by diabetes educators in the United States: one-year follow up. Diabetes Res Clin Pract 2014; 106:e49-52.

29. Kramer MK, Kriska AM, Venditti EM, et al. Translating the Diabetes Prevention Program: A comprehensive model for prevention training and program delivery. Am J Prev Med 2009;37:505-11.

30. Kramer MK, Kriska AM, Venditti EM, et al. A novel approach to diabetes prevention: evaluation of the Group Lifestyle Balance program delivered via DVD. Diabetes Res Clin Pract 2010;90:e60-63.

31. Kramer MK, Miller R, Venditti E, Orchard TO. Group lifestyle intervention for diabetes prevention in those with metabolic syndrome in primary care practice. Diabetes Care. 2006;55:A517.

32. McTigue KM, Conroy MB, Bigi L, Murphy C, McNeil M. Weight loss through living well: Translating an effective lifestyle intervention into clinical practice. Diabetes Educ 2009;35:199-204, 208.

33. Kramer MK, McWilliams JR, Chen HY, Siminerio LM. A community-based diabetes prevention program: Evaluation of the group lifestyle balance program delivered by diabetes educators. Diabetes Educ 2011;37:659-68.

34. Centers for Disease Control and Prevention Diabetes Prevention Recognition Program, Standards and Operating Procedures. In. Atlanta GA: Centers for Disease Control and Prevention; 2018. Available from: https://www.cdc.gov/diabetes/prevention/pdf/ dprp-standards.pdf.

35. Prochaska JO, DiClemente CC. Stages and processes of self-change of smoking: toward an integrative model of change. J Consult Clin Psychol 1983; 51:390-5.

36. Diabetes Prevention Support Center. NEW! 2017 DPP Group Lifestyle Balance Curriculum Information. 2017. Available from: http://www.diabetes prevention.pitt.edu/index.php/dpp-group-lifestyle- balance-curriculum-information/. Accessed December 1, 2017.

37. Hall DL, Lattie EG, McCalla JR, Saab PG. Translation of the Diabetes Prevention Program to Ethnic Communities in the United States. J Immigr Minor Health 2015;18:479-89.

38. Bowen G. Naturalistic inquiry and the saturation concept: A research note. Qual Res 2008;8:137-52.

39. Diagnosis and classification of diabetes mellitus. Diabetes Care. 2011;341:S62-9.

40. Grundy SM, Cleeman JI, Daniels SR, et al. Diagnosis and management of the metabolic syndrome: An American Heart Association/National Heart, Lung, and Blood Institute scientific statement. Circulation 2005;112:2735-52.

41. Chang SH, Yu YC, Carlsson NP, Liu X, Colditz GA. Racial disparity in life expectancies and life years lost associated with multiple obesity-related chronic conditions. Obesity (Silver Spring) 2017; 25:950-7.

42. Leeman-Castillo B, Beaty B, Raghunath S, Steiner J, Bull S. LUCHAR: Using computer technology to battle heart disease among Latinos. Am J Public Health 2010;100:272-5.

43. Marcus BH, Hartman SJ, Larsen BA, et al. Pasos Hacia La Salud: A randomized controlled trial of an internet-delivered physical activity intervention for Latinas. Int J Behav Nutr Phys Act 2016;13:62.

44. Benitez TJ, Cherrington AL, Joseph RP, et al. Using web-based technology to promote physical activity in latinas: Results of the Muevete Alabama pilot study. Comput Inform Nurs 2015;33:315-24.

45. Sarasohn-Kahn J. How smartphones are changing health care for consumers and providers. California HealthCare Foundation; 2010. Available from: https://www.chcf.org/publication/how-smartphonesare-changing-health-care-for-consumers-andproviders/.

46. Fox S, Duggan M. Health online 2013. 2013. Available from: http://www.pewinternet.org/2013/01/15/ health-online-2013/. Accessed on June 29, 2017.

47. Fox S, Duggan M. Mobile health 2012. 2012. Available from: http://pewinternet.org/Reports/2012/ Mobile-Health.aspx. Accessed on June 29, 2017.

48. Ayala GX, Baquero B, Klinger S. A systematic review of the relationship between acculturation and diet among Latinos in the United States: Implications for future research. J Am Diet Assoc 2008;108:1330-44.

49. Lara M, Gamboa C, Kahramanian MI, Morales LS, Bautista DE. Acculturation and Latino health in the United States: A review of the literature and its sociopolitical context. Annu Rev Public Health 2005; 26:367-97.

50. Berrigan D, Dodd K, Troiano RP, Reeve BB, Ballard-Barbash R. Physical activity and acculturation among adult Hispanics in the United States. Res Q Exerc Sport 2006;77:147-57. 